\title{
Propagation Characteristics of Fasting Duodeno- Jejunal Contractions in Healthy Controls Measured by Clustered Closely-spaced Manometric Sensors
}

\author{
Jason R Baker, ${ }^{1}$ Joseph R Dickens, ${ }^{2}$ Mark Koenigsknecht, ${ }^{2}$ Ann Frances, ${ }^{2}$ Allen A Lee, ${ }^{1}$ Kerby A Shedden, ${ }^{2}$ James G Brasseur, ${ }^{3}$ \\ Gordon L Amidon, ${ }^{2}$ Duxin Sun, ${ }^{2}$ and William L Hasler ${ }^{1 *}$
}

${ }^{I}$ Division of Gastroenterology, University of Michigan Health System, Ann Arbor, MI, USA; ${ }^{2}$ School of Pharmacy, University of Michigan, Ann Arbor, MI, USA; and ${ }^{3}$ Department of Aerospace Engineering Sciences, University of Colorado Boulder, Boulder, CO, USA

\section{Background/Aims}

High-resolution methods have advanced esophageal and anorectal manometry interpretation but are incompletely established for intestinal manometry. We characterized normal fasting duodeno-jejunal manometry parameters not measurable by standard techniques using clustered closely-spaced recordings.

\section{Methods}

Ten fasting recordings were performed in 8 healthy controls using catheters with 3-4 gastrointestinal manometry clusters with 1-2 $\mathrm{cm}$ channel spacing. Migrating motor complex phase III characteristics were quantified. Spatial-temporal contour plots measured propagation direction and velocity of individual contractions. Coupling was defined by pressure peak continuity within clusters.

\section{Results}

Twenty-three phase III complexes (11 antral, 12 intestinal origin) with 157 (95\% Cl, 104-211) minute periodicities, 6.99 (6.25-7.74) minute durations, 10.92 (10.68-11.16) cycle/minute frequencies, 73.6 (67.7-79.5) mmHg maximal amplitudes, and 4.20 (3.18$5.22) \mathrm{cm} /$ minute propagation velocities were recorded. Coupling of individual contractions was $39.1 \%(32.1-46.1) ; 63.0 \%$ (54.471.6) of contractions were antegrade and $32.8 \%$ (24.1-41.5) were retrograde. Individual phase III contractions propagated $>35$ fold faster $(2.48 \mathrm{~cm} / \mathrm{sec} ; 95 \% \mathrm{Cl}, 2.25-2.71)$ than complexes themselves. Phase III complexes beyond the proximal jejunum were longer in duration $(P=0.025)$ and had poorer contractile coupling $(P=0.025)$ than proximal complexes. Coupling was greater with $1 \mathrm{~cm}$ channel spacing vs $2 \mathrm{~cm}(P<0.001)$.

\section{Conclusions}

Intestinal manometry using clustered closely-spaced pressure ports characterizes novel antegrade and retrograde propagation and coupling properties which degrade in more distal jejunal segments. Coupling is greater with more closely-spaced recordings. Applying similar methods to dysmotility syndromes will define the relevance of these methods.

(J Neurogastroenterol Motil 2019;25:100-112)

Key Words

Intestines; Manometry; Migrating; Muscle contraction; Myoelectric complex

Received: June 18, 2018 Revised: October 22, 2018 Accepted: November 5, 2018

(.) This is an Open Access article distributed under the terms of the Creative Commons Attribution Non-Commercial License (http://creativecommons. org/licenses/by-nc/4.0) which permits unrestricted non-commercial use, distribution, and reproduction in any medium, provided the original work is properly cited.

*Correspondence: William L Hasler, MD

University of Michigan Health System, 3912 Taubman Center, SPC 5362, Ann Arbor, MI 48109, USA

Tel: +1-734-936-4780, Fax: +1-734-936-7392, E-mail: whasler@umich.edu 


\section{Introduction}

Small intestinal manometry is performed to exclude generalized dysmotility in patients with unexplained symptoms including nausea, vomiting, bloating, pain, and altered bowel function, or to distinguish neuropathic from myopathic patterns. ${ }^{1-3} \mathrm{~A}$ hallmark of manometric recording is detecting the migrating motor complex (MMC) which clears undigested residue during fasting; its electrical correlate, the migrating myoelectric complex, was first detected half a century ago. ${ }^{4}$ Interpreting intestinal manometry relies on defining qualitative factors like the presence of phase III MMC activity and induction of the fed pattern after consuming a caloric meal. ${ }^{2,5,6}$ Semiquantitative parameters can be gleaned from traditional intestinal manometry recordings in adults and children including phase III periodicity, antral versus duodenal site of phase III origin, phase III duration and frequency, phase III complex propagation velocity, and contractile amplitudes. ${ }^{7-19}$ As stated by others, clinical use of small bowel manometry has been limited by few measurement points with long distances between sensors. ${ }^{6} \mathrm{Nev}$ ertheless, use of such methods has had positive impacts on patient management in retrospective series including decisions to change medication treatments or nutritional regimens. ${ }^{20-22}$

High-resolution methods have advanced interpretation of esophageal and anorectal manometry. The Chicago classification has had major impact on high-resolution esophageal manometry including definitions of new diagnoses. ${ }^{23}$ However, less study has been devoted to intestinal manometry using similarly closelyspaced pressure sensors. Three studies employing catheters with $1.5 \mathrm{~cm}$ sensor spacing defined some propagation characteristics of individual phase III contractions in healthy volunteers. ${ }^{24-26}$ One study observed a high number of contractions which propagated in retrograde directions, while another noted bidirectional contractile propagation. ${ }^{24,26}$ Other properties relating to coupling of contractions have not been described and comparisons between duodenal versus jejunal phase III contractions have not been performed. Furthermore, the relation of sensor spacing to detection of propagation characteristics has not been assessed.

This investigation employed novel catheters with groupings of closely-spaced pressure ports that were positioned at sites from the duodenum to mid jejunum to perform fasting intestinal manometry in healthy controls. The study included post hoc analyses of fasting recordings from a parent study of ibuprofen distribution and absorption. Specific aims were to (1) quantify and compare phase III complex characteristics in relation to anatomic location and site of detected phase III origin, (2) characterize coupling of individual phase III contractions in the duodenum and jejunum as well as direction and velocities of propagation in each region, and (3) investigate the importance of pressure port spacing in quantifying coupling and propagation. These observations provide an assessment of manometric parameters over extended duodeno-jejunal segments using clusters of closely-spaced recordings in a healthy control cohort with implications for MMC physiology. Such findings may contribute to eventual standardization of quantitative fasting small intestinal motor activity measures to be subsequently evaluated by high-resolution manometry in patients with suspected generalized dysmotility.

\section{Materials and Methods}

\section{Subject Population}

Eight healthy volunteers (age 18 to 55 years, 4 women, 4 men) underwent 10 duodeno-jejunal manometry studies using catheters with clusters of closely-spaced pressure ports from August through November 2016 as a component of an investigation to examine dissolution and absorption of oral immediate release ibuprofen in relation to gastrointestinal transit and motility parameters (ClinicalTrials.gov NCT02806869). There were no differences with relation to subject sex in age (women: $35.7 \pm 9.5$ vs men: $28.4 \pm 5.4 ; P$ $=0.230$ ) or body mass index (women: $27.6 \pm 7.0$ vs men: $27.9 \pm$ $\left.7.2 \mathrm{~kg} / \mathrm{m}^{2} ; P=0.954\right)$. Medical history review and physical examination by study physicians (A.A.L. and W.L.H.) confirmed that all subjects were healthy without organic gastrointestinal disease, disorders of gut transit or motility, or gastrointestinal symptomatology. Laboratory tests (comprehensive chemistry panel, complete blood count, and lactate dehydrogenase), serum pregnancy tests (in women of child-bearing potential), electrocardiography, and urine drug screens (opiates, benzodiazepines, cannabinoids, amphetamines, cocaine, and barbiturates) were negative. Subjects were excluded if they were unable to provide informed consent, were mentally incapacitated, or had significant clinical illness in the 3 weeks prior to screening, medication use within the 2 weeks prior to testing (including prokinetics, antispasmodics, opiates, and calcium channel antagonists), or other conditions including cardiovascular, renal, hepatic, or gastrointestinal disorders that could, in the opinion of the investigators, jeopardize the safety of the patient or impact the validity of the study results.

Studies were approved by the Institutional Review Boards at University of Michigan Hospital (HUM00085066). Healthy 
subjects provided written informed consent prior to testing.

\section{Manometric Methods}

Multilumen catheters (Mui Scientific, Mississauga, ON, Canada) with 4 aspiration ports (20-30 cm spacing) and manometry pressure ports were employed for these studies. Each catheter was $292 \mathrm{~cm}$ in length with an outer diameter of $3.3 \mathrm{~mm}$, a central channel for a 0.035 in $\times 450 \mathrm{~cm}$ guidewire (Boston Scientific, Marlborough, MA, USA), another central channel connected to a deflated balloon ( $7 \mathrm{~mL}$ maximal volume) proximal to the tip, and a tungsten (7.75 g)-weighted tip. Two catheter designs with different pressure port spacing were included for this post-hoc investigation to relate manometry findings to resolution. Five manometry studies were performed in 4 volunteers using catheters with 3 groupings of 4 manometry ports spaced $1 \mathrm{~cm}$ apart beginning $2.5 \mathrm{~cm}$ proximal to the balloon near the catheter tip (distances $0-3 \mathrm{~cm}$ ), 4 ports beginning $42.5 \mathrm{~cm}$ proximal to the balloon (distances $40-43 \mathrm{~cm}$ ), and 4 ports beginning $67.5 \mathrm{~cm}$ proximal to the balloon (distances 65-68 $\mathrm{cm}$ ) (Fig. 1). Five other studies were performed in 4 subjects using catheters with 4 groupings of 3 manometry ports spaced $2 \mathrm{~cm}$ apart beginning $2.5 \mathrm{~cm}$ proximal to the balloon, 3 ports beginning 22.5 $\mathrm{cm}$ proximal to the balloon, 3 ports beginning $42.5 \mathrm{~cm}$ proximal to the balloon, and 3 ports beginning $67.5 \mathrm{~cm}$ proximal to the balloon.

Manometry catheter placement was performed after fasting for $>8$ hours. After swallowing topical anesthetic ( $1 \mathrm{~mL} 4 \%$ lidocaine), the catheter was orally introduced and initially passed under fluoroscopic guidance into the duodenum. The balloon near the tip was then inflated to $7 \mathrm{~mL}$ after initial duodenal catheter passage and the subject was allowed to ambulate for up to one hour to facilitate positioning in the mid jejunum. The balloon was subsequently deflated and the final catheter position was secured to a maximal depth in the mid jejunum $(>30 \mathrm{~cm}$ distal to the ligament of Treitz) for testing. The total time for catheter placement including the time of ambulation to facilitate advancement of the inflated balloon was $87.5 \pm 10.6$ minutes. The tube was connected to a perfusion pump which delivered water at about $100 \mathrm{~mL} / \mathrm{hr}$ through the 12 manometry ports and the subject was allowed to equilibrate for 1 hour prior to starting manometric recording. Fasting manometry (MMS Medical Measurement Systems, Williston, VT, USA) was performed for approximately 5 hours.

\section{Manometry Parameters}

Factors relating to duodeno-jejunal phase III complexes were characterized. All recording segments exhibiting evidence of motion artifacts from inadvertent subject movement were excluded from analysis. The fasting period was defined as the time from the end of the equilibration period to the time subjects consumed the test drug (ibuprofen) for the parent investigation, which was about 5 hours. Subjects remained awake for most of the fasting periods to minimize effects of sleep to alter phase III periodicity. ${ }^{9}$ Phase III complexes were visually identified using criteria similar to those previously described $^{11,24,26-29}$ : patterns of regular contractions at a frequency of
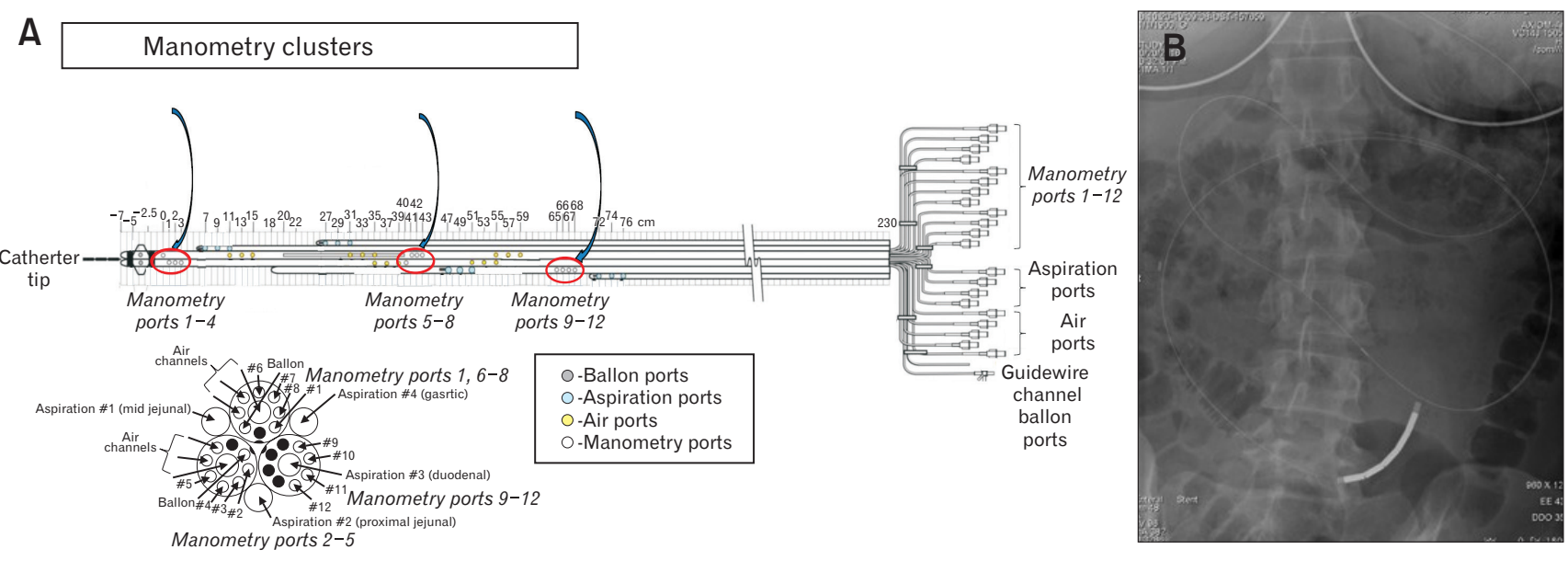

Figure 1. A schematic of the combined manometry and aspiration catheter with 3 clusters of 4 manometry ports (encircled in red ovals) with 1 $\mathrm{cm}$ spacing is shown (A). The catheter was designed as part of a parent study to study luminal medication absorption parameters and included additional ports to aspirate intestinal fluid (blue circles) and perfuse air during aspiration to optimize fluid recovery (yellow circles). A fluoroscopic image of a combined manometry and aspiration catheter with the tip in the mid jejunum is shown (B). Modified from Mui Scientific, Mississauga, ON, Canada with permission. 
10-12/minute lasting $\geq 2$ minutes with a minimal amplitude $\geq 10$ $\mathrm{mmHg}$ which propagated a distance of $\geq 3 \mathrm{~cm}$ and which were followed by a variable period of motor quiescence. The site of phase III origin was characterized as antral (regular 3 contraction/minute pattern for at least 2 minutes prior to duodenal phase III complex) or small intestinal. Small intestinal phase III complexes were further subdivided into those with detected origin in the duodenum or jejunum based on pressure port localization on fluoroscopy. Phase III cycling periodicity (minutes) was quantified by the number of duodeno-jejunal complexes divided by the duration of the fasting recording; the period of cycling is the inverse of periodicity. Phase III complexes not completed by the end of the fasting period were not included in this calculation. Phase III duration (minutes) was calculated from the first to the last contraction $>10 \mathrm{mmHg}$ during the complex, while contractile frequency per minute was defined by the total number of contractions $>10 \mathrm{mmHg}$ divided by the complex duration. This calculated contractile frequency is close to the standard reported cycling frequency of small bowel pacemaker activity; it is slightly less because our analyses excluded contractions that dropped out or were $<10 \mathrm{mmHg}$ in amplitude. ${ }^{29}$ Phase III propagation velocities within manometry clusters $(\mathrm{cm} / \mathrm{min})$ were calculated by dividing the $3-4 \mathrm{~cm}$ distance between the first and last pressure ports within a cluster by the time difference from the onset of the phase III complex in the first and last port. Propagation velocity between manometry clusters $(\mathrm{cm} / \mathrm{min})$ was calculated by dividing the distance between the first pressure port of one cluster and the first pressure port of the next cluster by the time of onset of phase III complexes in these ports. Maximal amplitude of contractions $(\mathrm{mmHg}$ ) was calculated for each duodeno-jejunal complex.

We also quantified measures of individual duodeno-jejunal phase III contractions. False color spatial-temporal contour plots were generated for each duodeno-jejunal phase III complex with a pressure range from $-17 \mathrm{mmHg}$ to $50 \mathrm{mmHg}$. All determinations of coupling were made by the senior corresponding author (W.L.H.). All phase III complexes were reviewed on 3 separate occasions in random order to ensure consistency of interpretation. Intra-observer variability was $<5 \%$; in rare cases where discrepancies in interpretation were seen, coupling was calculated from the mean of the 3 readings. Coupling of individual phase III contractions over an entire manometry pressure port cluster was defined if pressure peaks $>10 \mathrm{mmHg}$ were visually observed to be continuous without gaps from the first to the last pressure port over $3 \mathrm{~cm}$ (for $1 \mathrm{~cm}$ port spacing) or $4 \mathrm{~cm}$ (for $2 \mathrm{~cm}$ port spacing) distances within each sensor in the cluster. Because the shorter clusters of the catheters with $1 \mathrm{~cm}$ port spacing could yield apparently greater coupling values than the longer clusters of the catheters with $2 \mathrm{~cm}$ spacing, a second calculation of coupling percentages over the first $2 \mathrm{~cm}$ of each cluster with both catheter designs was performed. Percentages of individual coupled contractions within each phase III complex that propagated in an antegrade or retrograde direction or were stationary (no time difference between peaks in the first vs last ports within a pressure port cluster) were calculated. Propagation velocities of coupled antegrade and retrograde contractions $(\mathrm{cm} /$ $\mathrm{sec}$ ) showing coupling over the entire clusters within each phase III complex were calculated by dividing the distance from first to last pressure ports within a grouping by the time to each pressure peak on spatial-temporal contour plots.

\section{Subgroup Analyses}

Subgroup analyses assessed factors which influenced duodenojejunal MMC phase III complexes. Phase III duration, contractile frequency, propagation velocity within manometry clusters, propagation velocity between manometry clusters, and maximal contractile amplitude were compared in women versus men, for complexes located $>30 \mathrm{~cm}$ distal to the ligament of Treitz versus more proximally, for complexes originating in the antrum versus small intestine, and for $1 \mathrm{~cm}$ versus $2 \mathrm{~cm}$ manometry port spacing.

Subgroup analyses also evaluated factors affecting coupling of individual duodeno-jejunal phase III contractions and the direction of coupled phase III contractions. Percent coupling over entire manometry clusters and over the first $2 \mathrm{~cm}$ of each cluster was compared in women versus men, proximal versus distal complex location, antral versus small intestinal site of complex origin, and 1 $\mathrm{cm}$ versus $2 \mathrm{~cm}$ port spacing. Percentages of individual contractions coupled over entire manometry clusters that propagated antegrade versus retrograde versus stationary were compared for the same parameters. Given the findings of others of differences in direction of propagation in early versus late portions of the phase III complex, coupling parameters and direction of propagation were compared for individual contractions in the first half of each phase III complex (measured as the first $50 \%$ of contractions $>10 \mathrm{mmHg}$ ) versus the last half of each phase III complex. ${ }^{24,26,30}$

\section{Statistical Methods}

All data are expressed as number (n) and percent (\%) or mean with $95 \%$ CI. Two-tailed Student's $t$ testing was employed to determine relations of duodeno-jejunal phase III complex parameters (duration, contractile frequency, propagation velocity within and between phase III complexes, and maximal contractile amplitude) to sex (biological female vs male), anatomic location of phase III com- 
plex (more proximal vs $>30 \mathrm{~cm}$ distal to ligament of Treitz), site of phase III complex origin (antral vs small intestinal), and spacing of manometry pressure ports $(1 \mathrm{~cm}$ vs $2 \mathrm{~cm})$. Two tailed Student's $t$ testing also was performed to relate percent coupling over each entire cluster and the first $2 \mathrm{~cm}$ of each cluster and propagation velocities of individual phase III contractions to the same manometric factors plus early (1st half) versus late (2nd half) of each phase III complex. Two-way analysis of variance (ANOVA) was employed to relate percentages of propagated contractions over entire clusters that were antegrade, retrograde, or stationary to these manometric factors. Multiple logistic regression was applied to assess independent relationships of characteristics associated with coupling of individual phase III contractions measured both across entire manometry clusters and the first $2 \mathrm{~cm}$ of each cluster including: sex (male vs female), location of phase III complex (more proximal vs $>30$ $\mathrm{cm}$ distal to the ligament of Treitz), site of phase III origin (antral vs small bowel), early versus late phase III (first vs second half), and manometry port spacing $(1 \mathrm{~cm}$ vs $2 \mathrm{~cm})$. Statistical significance was defined by $P$-values of $<0.05$.

\section{Results}

\section{Characteristics of Duodeno-Jejunal Phase III Complexes}

Twenty-three duodeno-jejunal phase III complexes were recorded in 10 studies in 8 healthy volunteers. The mean duration of fasting recordings was 5.07 (95\% CI, 4.80-5.35) hours. No complications were observed from these studies other than minor throat irritation. Eleven phase III complexes (47.8\%) originated in the stomach and $12(52.2 \%)$ in the small intestine. Of those starting in the small bowel, 10/12 (83.3\%) initiated in the duodenum and
2/12 (16.7\%) were detected first by jejunal sensors. Two duodenojejunal phase III complexes (8.7\%) failed to propagate to the most distal recording channel. Figure 2 shows a representative phase III complex measured using a manometry catheter with 4 pressure port clusters with $2 \mathrm{~cm}$ spacing with origin in the antrum and sequential aboral propagation into the duodenum, proximal jejunum, and then mid jejunum.

\section{Subgroup Analyses of Factors Influencing Phase III Complex Characteristics}

Forty-six clusters of duodeno-jejunal phase III activity defined by the closely spaced manometry pressure ports were compared in the different small intestinal regions (Table 1A). On average, phase III complexes occurred roughly every 2 and a half hours, lasted 7 minutes, and propagated distally within and between manometry clusters at approximately $4 \mathrm{~cm}$ per minute. Contractile frequencies were approximately 11 cycles/minute and maximal contractile amplitudes were greater than $70 \mathrm{mmHg}$.

Factors relating to phase III activity were assessed (Table 1B). Maximal amplitude trended higher in men than women $(P=$

Table 1. Characteristics of Phase III Complexes A. Overall Characteristics of Phase III Complexes

\begin{tabular}{lc}
\hline \multicolumn{1}{c}{ Parameter } & Value (mean [95\% CI]) \\
\hline Periodicity (min) & $157(104-211)$ \\
Duration (min) & $6.99(6.25-7.74)$ \\
Contractile frequency (cycles/min) & $10.92(10.68-11.16)$ \\
Propagation velocity of complexes $(\mathrm{cm} / \mathrm{min})-$ & $4.20(3.18-5.22)$ \\
$\begin{array}{l}\text { within clusters } \\
\text { Propagation velocity of complexes }(\mathrm{cm} / \mathrm{min})-\end{array}$ & $3.72(2.82-4.56)$ \\
$\quad$ between clusters & \\
Maximal contractile amplitude $(\mathrm{mmHg})$ & $73.6(67.7-79.5)$ \\
\hline
\end{tabular}

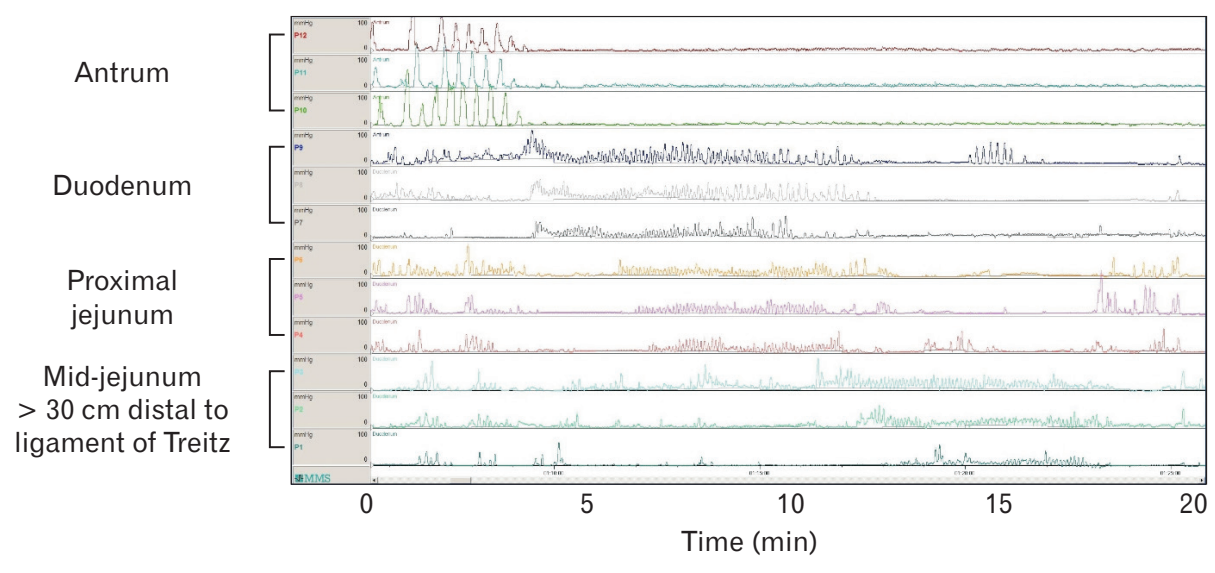

Figure 2. A representative phase III complex measured using a catheter with 4 clusters of 3 manometry ports at $2 \mathrm{~cm}$ intervals with the complex originating in the antrum (ports 1-3) and propagating through the duodenum (ports 4-6), proximal jejunum (ports 7-9), and mid jejunum (ports 10-12) is shown. 


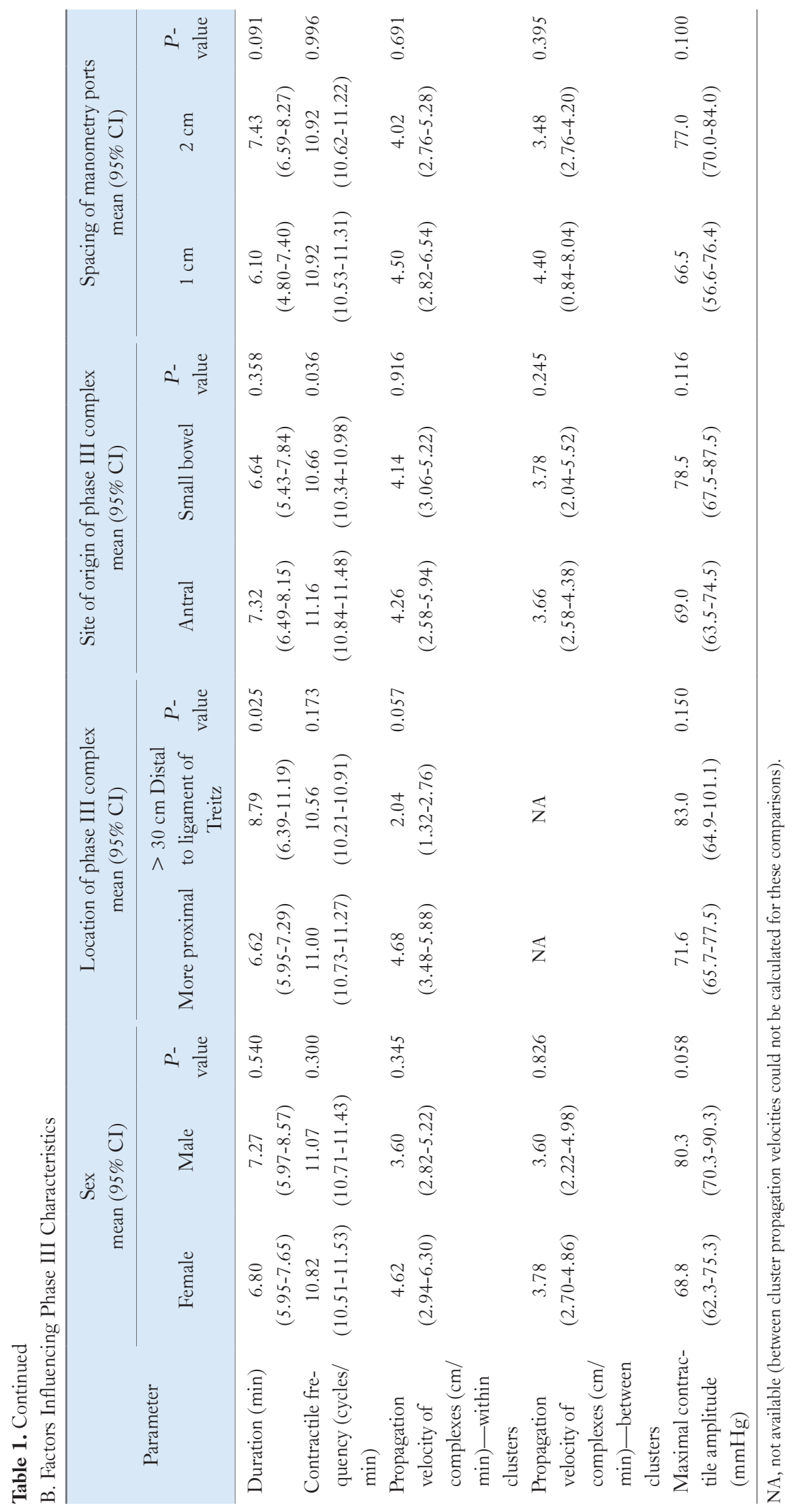


0.058). Complexes $>30 \mathrm{~cm}$ distal to the ligament of Treitz were longer in duration $(P=0.025)$ and trended to slower propagation within manometry clusters $(P=0.057)$ versus in the duodenum or proximal jejunum. Contractile frequencies were greater for complexes originating in the antrum versus in the small bowel $(P=$ 0.036). Pressure port spacing did not relate to any phase III complex measure.

\section{Characteristics of Individual Phase III Contractions}

Characteristics of individual phase III contractions are shown in Table 2. We found that 39.1\% (95\% CI, 32.1-46.1) of individual contractions were coupled over the 3-4 cm distance of each manometry cluster, but $61.1 \%$ (95\% CI, 53.0-69.2) propagated the first 2

Table 2. Characteristics of Individual Phase III Contractions

\begin{tabular}{lc}
\hline \multicolumn{1}{c}{ Parameter } & $\begin{array}{c}\text { Value } \\
\text { mean (95\% CI) }\end{array}$ \\
\hline $\begin{array}{l}\text { Coupling of contractions_-across entire } \\
\text { clusters }\end{array}$ & $39.1 \%(32.1-46.1)$ \\
Coupling of contractions_over the first 2 & $61.1 \%(53.0-69.2)$ \\
cm of each cluster & \\
$\%$ of coupled contractions_-antegrade & $63.0 \%(54.4-71.6)$ \\
$\%$ of coupled contractions_-retrograde & $32.8 \%(24.1-41.5)$ \\
$\%$ of coupled contractions_-stationary & $4.2 \%(1.5-6.9)$ \\
Propagation velocity $(\mathrm{cm} / \mathrm{sec})$ - antegrade & $2.35(2.00-2.70)$ \\
contractions & \\
Propagation velocity $(\mathrm{cm} / \mathrm{sec})$ - retrograde & $2.63(2.36-2.90)$ \\
contractions & \\
\hline
\end{tabular}

$\mathrm{cm}$ of each cluster. Of all contractions coupled over the entire 3-4 cm cluster distance, $63.0 \%$ (95\% CI, 54.4-71.6) were antegrade while $32.8 \%$ (95\% CI, 24.1-41.5) propagated in a retrograde direction (Fig. 3). Propagation velocities of individual contractions were much greater than velocities of propagation of phase III complexes themselves $(P<0.001)$.

\section{Subgroup Analyses of Factors Influencing Individual Phase III Contraction Coupling}

Factors influencing coupled individual contractions were evaluated. Percent of phase III contractions that were coupled over entire clusters $(P<0.001)$ and over the first $2 \mathrm{~cm}$ of each cluster $(P=$ 0.029 ) were higher in women versus men (Fig. 4A). The percent of individual contractions showing coupling over the first $2 \mathrm{~cm}$ of each cluster $(P=0.025)$ but not entire clusters was lower for complexes $>30 \mathrm{~cm}$ distal to the ligament of Treitz versus more proximal complexes (Fig. 4B). Coupling of phase III contractions over entire clusters $(P<0.001)$ and over the first $2 \mathrm{~cm}$ of each cluster $(P=$ 0.003 ) from studies which used manometry catheters with $1 \mathrm{~cm}$ spacing between pressure ports was significantly greater than contractions measured by catheters with $2 \mathrm{~cm}$ manometry port spacing (Fig. 4E). Site of phase III origin and early versus late portion of phase III complexes did not influence either parameter of coupling of individual contractions ( $P=\mathrm{NS}$ ) (Fig. 4C and 4D).

Factors contributing to propagation of individual phase III contractions were assessed. The percent of coupled contractions that propagated over entire manometry clusters in antegrade versus retrograde direction or were stationary were similar in relation to
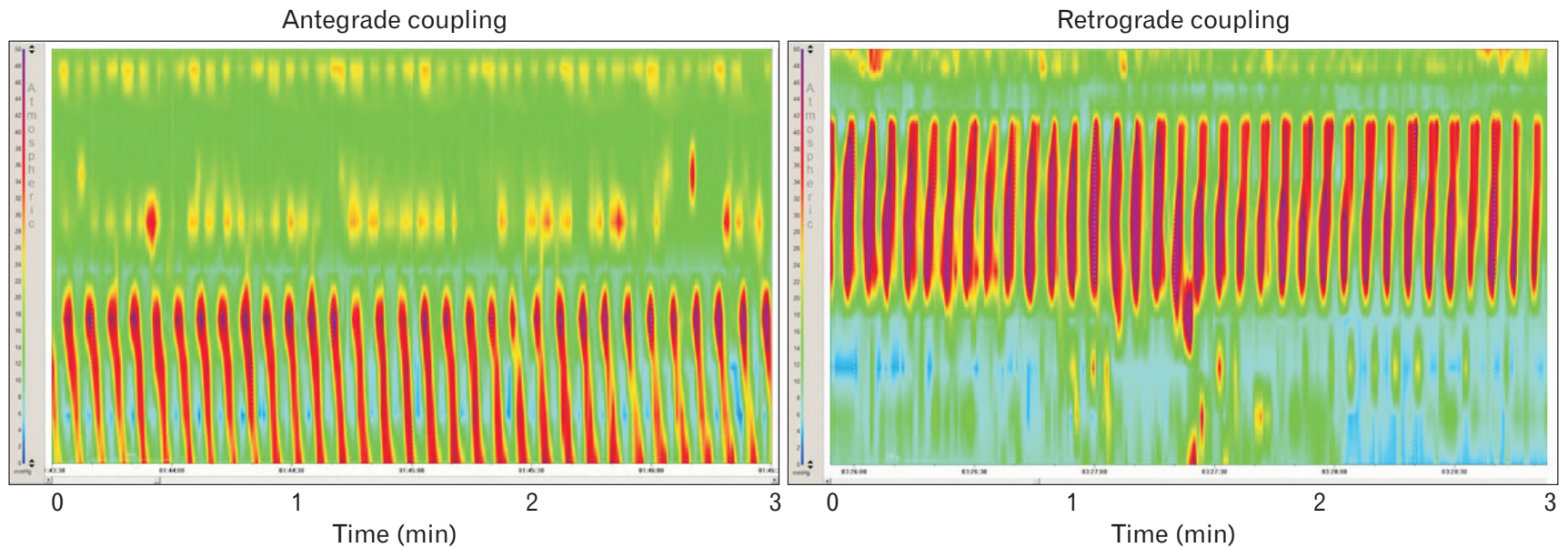

Figure 3. The figure on the left shows an example of antegrade coupling of contractions within a proximal jejunal phase III complex. The figure on the right shows retrograde coupling of contractions within a duodenal phase III complex. In each of these figures, the coupled contractions show continuity of the red-colored pressure contours $>25 \mathrm{mmHg}$ across the $3 \mathrm{~cm}$ distance of the manometry port clusters. 
A

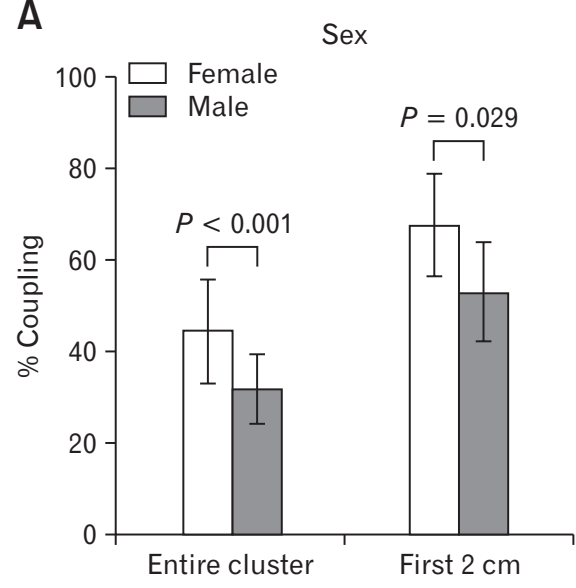

D

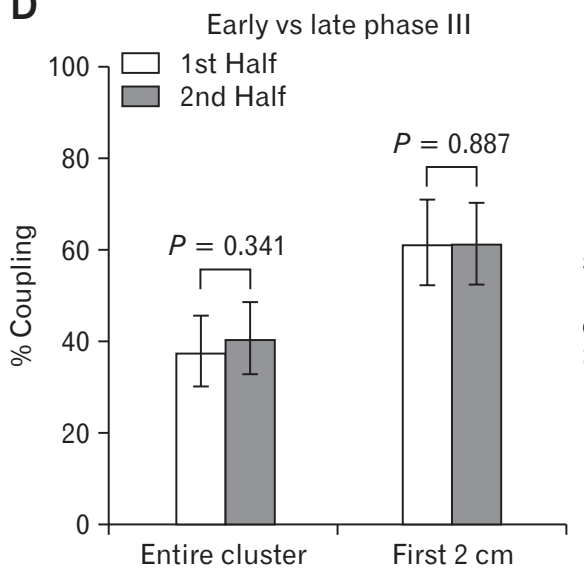

B

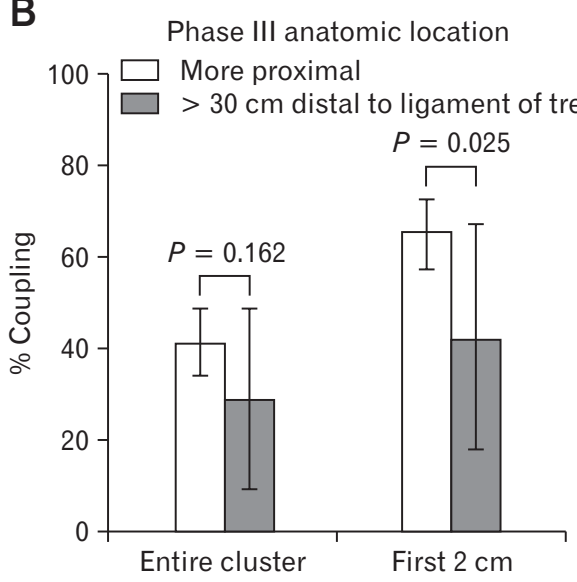

E

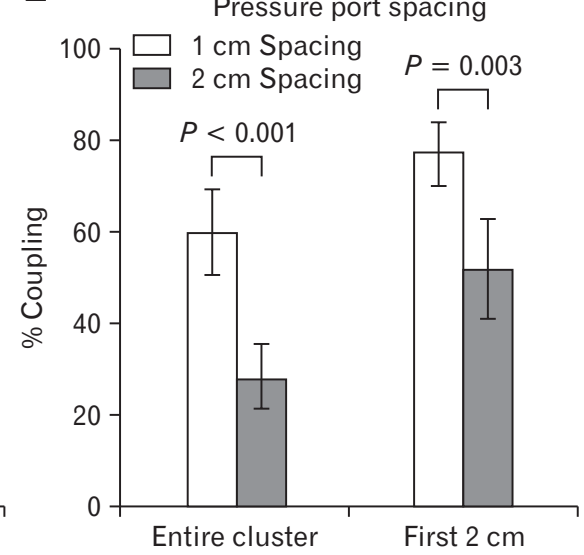

C

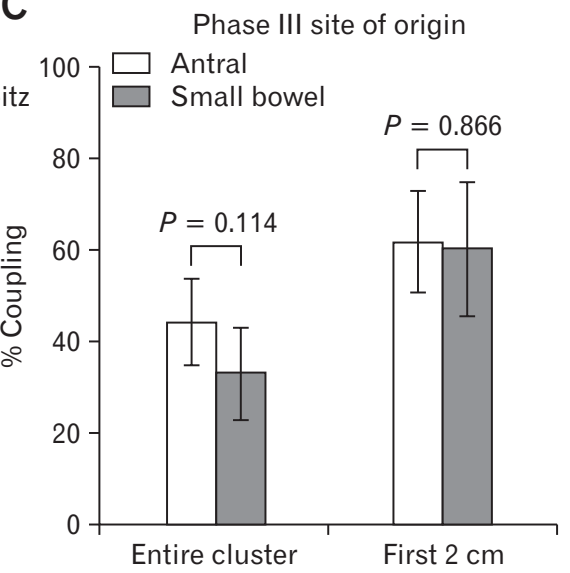

Figure 4. Subgroup analyses defined factors which influenced coupling of individual phase III contractions over the entire manometry port cluster and over the first $2 \mathrm{~cm}$ of each cluster. The percent of coupled contractions was greater in women than men for both the entire cluster and the first $2 \mathrm{~cm}$ of each cluster (A). The percent coupling over the first $2 \mathrm{~cm}$ of each cluster from more proximal phase III complexes was significantly higher vs $>30 \mathrm{~cm}$ distal to the ligament of Treitz; the percent coupling for the entire cluster was not different for proximal vs distal complexes (B). The percent of coupled contractions from complexes recorded with $1 \mathrm{~cm}$ manometry port spacing was higher vs $2 \mathrm{~cm}$ manometry port spacing for both the entire clusters and the first $2 \mathrm{~cm}$ of each cluster (E). Site of phase III origin (C) and early vs late in phase III (D) did not influence coupling of individual phase III contractions measured by either parameter.

sex, phase III complex location, site of phase III complex origin, and early versus late phase III ( $P=$ NS) (Table 3A). There was a greater percentage of antegrade and lower percentage of retrograde contractions recorded by catheters with $1 \mathrm{~cm}$ versus $2 \mathrm{~cm}$ manometry port spacing $(P=0.003)$. Propagation velocities of antegrade and retrograde coupling contractions did not show differences in women versus men, antral versus small intestinal phase III origin, early versus late phase III, and $1 \mathrm{~cm}$ versus $2 \mathrm{~cm}$ port spacing $(P=$ NS) (Table 3B). Retrograde contractions from phase III complexes originating in the antrum propagated faster than those from complexes starting in the small bowel $(P=0.003)$.

\section{Multiple Logistic Regression}

Multiple regression analyses defined factors relating to coupling of individual phase III contractions measured across entire clusters and the first $2 \mathrm{~cm}$ of each cluster (Table 4). Sensor spacing of $1 \mathrm{~cm}$ was associated with increased coupling over entire clusters $(P=0.012)$, while antral phase III origin trended to increased coupling $(P=0.068)$. These factors were not associated with increased coupling over the first $2 \mathrm{~cm}$ of each cluster.

\section{Discussion}

This study used novel catheters that permitted detailed duode- 

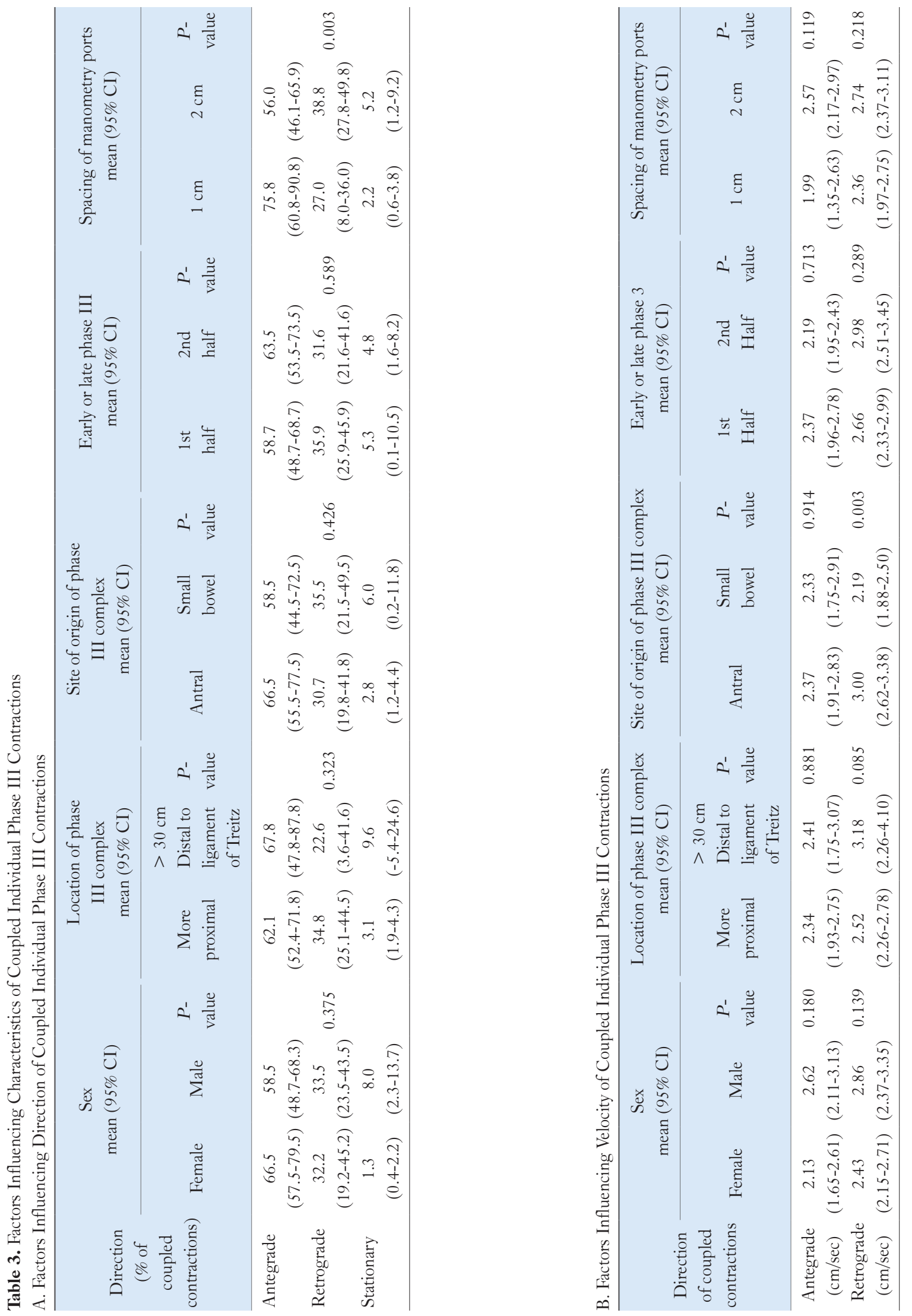
Table 4. Multiple Regression Analyses to Define Factors Relating to Coupling of Individual Phase III Contractions

\begin{tabular}{lcccc}
\multicolumn{1}{c}{ Parameter } & \multicolumn{2}{c}{ Entire cluster } & & \multicolumn{2}{c}{ First $2 \mathrm{~cm}$ of each cluster } \\
\cline { 2 - 3 } & Odds ratio (95\% CI) & $P$-value & Odds ratio (95\% CI) & $P$-value \\
\hline Female sex & $1.03(0.98-1.08)$ & 0.220 & $1.00(0.97-1.04)$ \\
Location $>$ 30 cm distal to ligament of Treitz & $1.01(0.94-1.09)$ & 0.769 & $0.96(0.90-1.02)$ & 0.192 \\
Antral origin & $1.04(1.00-1.10)$ & 0.068 & $0.97(0.94-1.01)$ & 0.182 \\
Early phase III (first half) & $0.99(0.96-1.01)$ & 0.332 & $1.01(0.99-1.03)$ & 0.440 \\
1 cm Manometry port spacing & $1.10(1.02-1.78)$ & 0.012 & $0.99(0.93-1.05)$ & 0.780 \\
\hline
\end{tabular}

no-jejunal recordings in healthy humans. Pressure port groupings allowed measurement of extended contractile activity over $>60 \mathrm{~cm}$ bowel segments and scrutiny of local propagation characteristics from closely-spaced sensors within each port grouping.

Our phase III complex quantifications complement prior reports. The $48 \%$ rate of antral complex origin, 157 minute intervals between complex onset, 10.92 cycles/min contractile frequencies, and $3.72-4.68 \mathrm{~cm} / \mathrm{min}$ propagation velocities are similar to previous values. ${ }^{7,8,11-15,17-19,26,31}$ Our 6.99 minute complex durations were slightly longer than prior studies. ${ }^{8,14-19,24,26,31}$

This study provides new information on factors affecting phase III morphology. We found no sex differences in periodicity, duration, contractile frequency, or propagation velocity, in contrast to a study which noted reduced phase III periodicity and duration in women. ${ }^{9}$ Another group found longer periods and durations in complexes of antral versus duodenal origin. ${ }^{15}$ We observed longer complex durations $>30 \mathrm{~cm}$ distal to the ligament of Treitz. Longer phase III duration and reduced frequencies have been seen in human ileum. ${ }^{31}$

Adopting closely-spaced sensors permitted quantifying individual contraction propagation. Most prior methods used 3-15 cm spacing which cannot detect propagation of individual contractions. ${ }^{11,17,19,27-29,31-33}$ Two studies using 1-2 cm spaced sensors did not measure propagation. ${ }^{12,15}$ In a third report, phase III contraction origins measured every $1.5 \mathrm{~cm}$ migrated $8 \mathrm{~cm}$ distally from the proximal duodenum as the complex progressed. ${ }^{25}$ Two thirds of proximal duodenal contractions propagated in retrograde direction in a fourth study using $1.5 \mathrm{~cm}$ spacing versus $17-25 \%$ in the distal duodenum. ${ }^{24}$ Our intermediate retrograde percentage of $34.8 \%$ may be due to more dedicated proximal duodenal recordings by the other group. ${ }^{24}$ In contrast to our study, they also noted greater distal duodenal retrograde contractions in the latter $(75 \%)$ versus early $(25 \%)$ complex phase. A fifth examination using $1.5 \mathrm{~cm}$ sensor spacing observed frequent bidirectional propagation with bifurcation of individual contractions into proximally- and distally- propagating components. ${ }^{26}$ We likely did not observe these bifurcations because of the short lengths of the manometry port clusters.

Some studies determined propagation using hand drawn techniques. One study delineated waveform direction as "manually marked" while hand drawn propagation lines were noted in another study's figures. ${ }^{24,25}$ We applied false color spatial-temporal contour plots to identify coupling and direction of contractile propagation. Similar methods have been applied in high-resolution esophageal manometry and by one other high-resolution duodenal manometry study. ${ }^{26,34}$ One can argue that automated isobaric measurements minimize bias in defining direction of coupled contractions.

Parameters not previously evaluated using closely-spaced sensors included percent antegrade and retrograde coupling and relating coupling and directionality to sex, complex location, site of origin, and early versus late phase III. Coupling was greater in women and distal complexes on univariate analyses. The lack of significance on regression testing suggests other factors influenced these findings. Propagation velocities of antegrade contractions in this study were similar to prior reports. ${ }^{26,31}$ Contractile velocities were faster in complexes with antral origin.

These analyses offer insight into differential control of phase III complexes and individual contractions within each complex. Propagation velocities of individual contractions were $>35$-fold greater than velocities of complexes. Thus, most individual contractions propagated only a short fraction of the distance each phase III complex migrated. Early phase III contractions did not propagate over the full cluster distance because they "outran" the complex. No retrograde phase III complexes were seen even though one third of individual contractions were retrograde. One quarter of complexes were comprised of mostly retrograde contractions; 2 complexes had only retrograde contractions. It is possible that interactions of divergent extended long-distance and local short-distance motor activities promote aboral propulsion of undigested gut contents with regional mixing to facilitate mucosal absorption of gut contents.

A striking finding is the greater coupling of individual contrac- 
tions using $1 \mathrm{~cm}$ versus $2 \mathrm{~cm}$ port spacing, likely reflecting greater sensitivity of more closely-spaced sensors. Because of limitations imposed on catheter design by the parent study, cluster length was $3 \mathrm{~cm}$ for $1 \mathrm{~cm}$ spaced tubes versus $4 \mathrm{~cm}$ for $2 \mathrm{~cm}$ spaced catheters. We were initially concerned that lower coupling found with $2 \mathrm{~cm}$ spacing might be due to large numbers of contractions that propagated $<4 \mathrm{~cm}$. However, this concern was dispelled by prior reports documenting $8.6 \mathrm{~cm}$ antegrade and $\geq 4 \mathrm{~cm}$ retrograde propagation distances. ${ }^{25,31}$ Additional analyses defined greater coupling detection with $1 \mathrm{~cm}$ versus $2 \mathrm{~cm}$ spacing over the first $2 \mathrm{~cm}$ of each cluster, a common distance for both catheter designs, confirming enhanced capabilities of closely-spaced sensors. Given the greater fidelity recordings from $1 \mathrm{~cm}$ spaced catheters, we believe the higher percentage of antegrade contractions observed with closely-spaced pressure ports is more accurate.

This study had limitations. Our catheter designs allowed highresolution measurements within clusters over long distances, but much of the bowel was unevaluated including the pylorus and duodenal bulb. Thus, we did not assess antropyloroduodenal coordination or confirm prior reports of proximal duodenal retrograde contractions. ${ }^{24,25,30,35}$ The $3-4 \mathrm{~cm}$ cluster lengths could not quantify maximal distances of contractile propagation and could not detect bidirectional propagation for waveforms that migrate both proximally and distally. Others proposed recordings must exhibit typical complex morphologies for distances $>25 \mathrm{~cm}$ to be considered true phase III activity, a span not observed in all subjects in this study. ${ }^{26}$ Coupled contractions in our study had to show contractile amplitudes $\geq 10 \mathrm{mmHg}$, which may have artifactually excluded lower amplitude coupled contractions. However, the physiologic importance of low amplitude activity in interdigestive transit is unproved and other methods of measuring small bowel motor activity use $10 \mathrm{mmHg}$ cutoffs for contractile quantifications. ${ }^{36}$ Finally, we acknowledge the sample size of our study was small ( $\mathrm{n}=8$ subjects). This sample was restricted by the parent study which was funded to evaluate ibuprofen absorption in only a limited group of healthy individuals. This small sample also is relevant to our subgroup comparisons, such as between sexes, and mandates further studies in larger healthy control cohorts. We defined our sample sizes by numbers of phase III complexes rather than subject numbers because of intrasubject differences in phase III complex properties and individual propagation direction characteristics. Although this decision yielded greater $\mathrm{CI}$ for some parameters, we believe this reflects the diversity of phase III complex and individual contraction profiles in health.

Although local propagation characteristics of individual con- tractions were defined by our catheter designs, continuous longdistance high-resolution recordings were not generated like those from commercial esophageal catheters with 30 or more contiguous channels at about $1 \mathrm{~cm}$ intervals. Our catheters were restricted to 12 ports by constraints of the parent study. Nevertheless, a strength of our study is use of catheters with groupings of high-resolution sensors over long distances which could be used to characterize dysmotility over extended intestinal segments in patients with unexplained symptoms. This permitted recordings from the proximal duodenum to the mid jejunum-distances significantly greater than most standard manometry protocols. As one potential example of a clinical catheter design, a 30-channel catheter with 5 clusters of 6 pressure ports at $1 \mathrm{~cm}$ intervals could measure extended plus local contractile activity in high-resolution fashion over $60-90 \mathrm{~cm}$ of intestine as far as the mid-jejunum instead of the $25-30 \mathrm{~cm}$ distances in the duodenum using catheters without clustered sensors. ${ }^{24-26}$

While quantifying phase III contractile properties has relevance to intestinal motility, further research is needed to determine the clinical relevance of our findings. We focused on phase III contractions because this complex has the most stereotypical properties of any manometric parameter and diagnosis of small bowel dysmotility using clinical manometry depends on detecting phase III activity. In children with suspected dysmotility, detection of phase III activity plus fed responses to meals is sufficient to exclude a diagnosis of intestinal pseudoobstruction. ${ }^{16} \mathrm{~A}$ strength of our study is its calculation of $95 \% \mathrm{CI}$ of normal ranges of several parameters including duration and propagation velocities of phase III complexes in different regions and properties of individual contractions like coupling and direction and propagation velocities of coupled contractions. The clinical importance of contractile coupling is unknown. Phase III is the period of most rapid small bowel transit during fasting. ${ }^{37}$ Similarly, we observed higher coupling in phase III versus phase II of the MMC. ${ }^{38}$ Future studies will correlate coupling with transit and symptoms in patients with dysmotility syndromes. We did not examine propagation characteristics of fed motor contractions after eating due to inadequate fed testing with closely-spaced sensors. Patients with gastroparesis commonly report postprandial symptoms, but this relationship is less clear for small bowel dysmotility syndromes. ${ }^{39}$ However, loss of duodenal phase III in dysmotility syndromes is associated with development of small intestinal bacterial overgrowth demonstrating a pathogenic relevance of fasting impairments. ${ }^{40}$ Finally, it would be important to compare contractile coupling and propagation after prokinetic stimulation with agents such as erythromycin or octreotide in healthy controls and dysmotility patients to further determine if phase III parameters might be 
potentially correctable with pharmaceutical stimulation.

Clinical interpretation of intestinal manometry is usually performed in qualitative fashion. It is likely that patients with dysmotility will exhibit quantitative abnormalities of phase III contractile propagation detectable by sophisticated methods like those reported here. It is possible that individuals with mild enteric neuropathy but preserved phase III complexes might exhibit reduced coupling of individual contractions or altered propagation velocity or directionality. As an analogy, high-resolution esophageal manometry permits diagnosis of several conditions in the recent Chicago classification not detectable by older low-resolution methods including ineffective esophageal motility, esophagogastric junction outflow obstruction, and fragmented peristalsis. ${ }^{23}$ We recognize that other methods of manometric interpretation have been proposed and there is no gold standard for performance of small intestinal manometry. However, we believe that the combination of closely spaced sensors with clustered pressure ports permitting long distance recordings offers advantages over some other methods. Defining a gold standard will require detailed testing in dysmotility syndromes with input from experts in technical review publications.

In conclusion, manometry using catheter designs with clusters of closely-spaced sensors spanning extended distances characterizes novel parameters relating to antegrade and retrograde propagation and coupling of individual duodeno-jejunal phase III contractions which degrade in distal segments in addition to properties of phase III complexes themselves. Coupling is greater with $1 \mathrm{~cm}$ versus 2 $\mathrm{cm}$ recording port spacing. These observations further the process of standardizing quantitative measures of fasting duodeno-jejunal motor activity in health which will be compared to findings of future studies measuring high-resolution manometry in patients with unexplained upper gastrointestinal symptoms and suspected small intestinal dysmotility.

Financial support: The parent investigation supporting this study received funding from the United States Food and Drug Administration (Grant No. HHSF223201310144C).

\section{Conflicts of interest: None.}

Author contributions: Jason R Baker: study design, experimental conduct, data collection, data analysis, manuscript preparation, and approval of final manuscript version;

Joseph R Dickens: data analysis and approval of final manuscript version; Mark Koenigsknecht: study design, experimental conduct, data collection, and approval of final manuscript version;
Ann Frances: study design, experimental conduct, data collection, and approval of final manuscript version; Allen A Lee: experimental conduct, data collection, and approval of final manuscript version; Kerby A Shedden: data analysis, approval of final manuscript version; James G Brasseur: data analysis, approval of final manuscript version; Gordon L Amidon: study design, experimental conduct, funding procurement, and approval of final manuscript version; Duxin Sun: study design, experimental conduct, funding procurement, and approval of final manuscript version; William L Hasler: study design, experimental conduct, data collection, data analysis, manuscript preparation, and approval of final manuscript version; and all authors had access to study data and reviewed and approved the final manuscript.

\section{References}

1. Mearin F, Malagelada JR. Gastrointestinal manometry: a practical tool or a research technique? J Clin Gastroenterol 1993;16:281-291.

2. Bassotti G, Bologna S, Ottaviani L, Russo M, Dore MP. Intestinal manometry: who needs it? Gastroenterol Hepatol Bed Brench 2015;8:246252

3. Malagelada C, Malagelada JR. Small bowel motility. Curr Gastroenterol Rep 2017;19:26.

4. Szurszewski JH. A migrating electrical complex of the canine small intestine. Am J Physiol 1969;217:1757-1763.

5. Gronlund D, Poulsen JL, Sandberg TH, et al. Established and emerging methods for assessment of small and large intestinal motility. Neurogastroenterol Motil Published Oline First:13 Jan 2017;29. doi: 10.1111/ nmo. 13008

6. Lindberg G. High-resolution manometry changes our views of gastrointestinal motility. Neurogastroenterol Motil 2013;25:780-782.

7. Testoni PA, Fanti L, Bagnolo F, et al. Manometric evaluation of the interdigestive antroduodenal motility in subjects with fasting bile reflux, with and without antral gastritis. Gut 1989;30:443-448.

8. Quigley EM, Donovan JP, Lane MJ, Gallagher TF. Antroduodenal manometry. Usefulness and limitations as an outpatient study. Dig Dis Sci 1992;37:20-28.

9. Wilson P, Perdikis G, Hinder RA, Redmond EJ, Anselmino M, Quigley EM. Prolonged ambulatory antroduodenal manometry in humans. Am J Gastroenterol 1994;89:1489-1495.

10. Jebbink RJ, van Berge-Henegouwen GP, Akkermans LM, Smout AJ. Antroduodenal manometry: 24-hour ambulatory monitoring versus short-term stationary manometry in patients with functional dyspepsia. Eur J Gastroenterol Hepatol 1995;7:109-116.

11. Samsom M, Jebbink RJ, Akkermans LM, van Berge-Henegouwen GP, Smout AJ. Abnormalities of antroduodenal manometry in type 1 diabetes. Diabetes Care 1996;19:21-27.

12. Holland R, Gallagher MD, Quigley EM. An evaluation of an ambulatory manometry system in assessment of antroduodenal motor activity. Dig Dis Sci 1996;41:1531-1537. 
13. Tomomasa T, Di Lorenzo C, Morikawa A, Uc A, Hyman PE. Analysis of fasting antroduodenal manometry in children. Dig Dis Sci 1996;41:2195-2203.

14. Gielkens HA, Nieuwenhuizen A, Biemond I, Lamers CB, Masclee AA. Interdigestive antroduodenal motility and gastric acid secretion. Aliment Pharmacol Ther 1998;12:27-33.

15. Luiking YC, van der Reijden AC, van Berge Henegouwen GP, Akkermans LM. Migrating motor complex cycle duration is determined by gastric or duodenal origin of phase III. Am J Physiol 1998;275(6 Pt 1):G1246-G1251.

16. Cucchiara S, Borrelli O, Salvia G, et al. A normal gastrointestinal motility excludes chronic intestinal pseudoobstruction in children. Dig Dis Sci 2000;45:258-264.

17. Bortolotti M, Annese V, Coccia G. Twenty-four hour ambulatory antroduodenal manometry in normal subjects (co-operative study). Neurogastroenterol Motil 2000;12:231-238.

18. Haans JJ, Masclee AA. Intragastric acidification inhibits motilin-induced phase III activity in humans. Neurogastroenterol Motil 2006;18:637646.

19. Brun R, Michalek W, Surjanhata BC, Parkman HP, Semler JR, Kuo B. Comparative analysis of phase III migrating motor complexes in stomach and small bowel using wireless motility capsule and antroduodenal manometry. Neurogastroenterol Motil 2012;24:332-e165.

20. Byrne KG, Quigley EM. Antroduodenal manometry: an evaluation of an emerging methodology. Dig Dis 1997;15(suppl 1):53-63.

21. Verhagen MA, Samsom M, Jebbink RJ, Smout AJ. Clinical relevance of antroduodenal manometry. Eur J Gastroenterol Hepatol 1999;11:523528.

22. Soffer $\mathrm{E}$, Thongsawat $\mathrm{S}$. Clinical value of duodenojejunal manometry. Its usefulness in diagnosis and management of patients with gastrointestinal symptoms. Dig Dis Sci 1996;41:859-863.

23. Kahrilas PJ, Bredenoord AJ, Fox M, et al. The Chicago classification of esophageal motility disorders, v3.0. Neurogastroenterol Motil 2015;27:160-174.

24. Castedal M, Björnsson E, Abrahamsson H. Duodenal juxtapyloric retroperistalsis in the interdigestive state in humans. Scand J Gastroenterol 1997;32:797-804.

25. Castedal M, Abrahamsson H. High-resolution analysis of the duodenal interdigestive phase III in humans. Neurogastroenterol Motil 2001;13:473-481.

26. Andrews JM, O'donovan DG, Hebbard GS, Malbert CH, Doran SM, Dent J. Human duodenal phase III migrating motor complex activity is predominantly antegrade, as revealed by high-resolution manometry and colour pressure plots. Neurogastroenterol Motil 2002;14:331-338.

27. Samsom M, Jebbink RJ, Akkermans LM, Bravenboer B, vanBerge-
Henegouwen GP, Smout AJ. Effects of oral erythromycin on fasting and postprandial antroduodenal motility in patients with type 1 diabetes, measured with an ambulatory manometric technique. Diabetes Care 1997;20:129-134.

28. Uc A, Hoon A, Di Lorenzo C, Hyman PE. Antroduodenal manometry in children with no upper gastrointestinal symptoms. Scand J Gastroenterol 1997;32:681-685.

29. Glia A, Lindberg G. Antroduodenal manometry findings in patients with slow-transit constipation. Scand J Gastroenterol 1998;33:55-62.

30. Björnsson ES, Abrahamsson H. Interdigestive gastroduodenal manometry in humans. Indication of duodenal phase III as a retroperistaltic pump. Acta Physiol Scand 1995;153:221-230.

31. Seidl H, Gundling F, Pfeiffer A, Pehl C, Schepp W, Schmidt T. Comparison of small-bowel motility of the human jejunum and ileum. Neurogastroenterol Motil 2012;24:e373-e380.

32. Penning C, Gielkens HA, Hemelaar M, Lamers CB, Masclee AA. Reproducibility of antroduodenal manometry during prolonged ambulatory recording. Neurogastroenterol Motil 2001;13:133-141.

33. Moak JP, Fabian RR, Clarke LC, Hanumanthaiah S, Desbiens J, Darbari A. Antroduodenal manometry is abnormal in children presenting with orthostatic intolerance and gastrointestinal symptoms. J Pediatr Gastroenterol Nutr 2106;63:329-335.

34. Van Hoeij FB, Bredenoord AJ. Clinical application of esophageal highresolution manometry in the diagnosis of esophageal motility disorders. J Neurogastroenterol Motil 2016;22:6-13.

35. Mearin F, Camilleri M, Malagelada JR. Pyloric dysfunction in diabetics with recurrent nausea and vomiting. Gastroenterology 1986;90:19191925.

36. Hasler WL, May KP, Wilson LA, et al. Relating gastric scintigraphy and symptoms to motility capsule transit and pressure findings in suspected gastroparesis. Neurogastroenterol Motil Published Online First: 5 Sep 2017. doi: 10.1111/nmo.13196.

37. Kerlin P, Zinsmeister A, Phillips S. Relationship of motility to flow of contents in the human small intestine. Gastroenterology 1982;82:701706.

38. Hasler WL, Baker J, Dickens J, et al. Standardizing parameters of fasting duodenojejunal manometry in healthy volunteers using clustered high-resolution pressure ports: focus on migrating motor complex phase 2 contractions. Gastroenterology 2018;154:S-311.

39. Parkman HP, Hallinan EK, Hasler WL, et al. Nausea and vomiting in gastroparesis: similarities and differences in idiopathic and diabetic gastroparesis. Neurogastroenterol Motil 2016;28:1902-1914.

40. Vantrappen G, Janssens J, Hellemans J, Ghoos Y. The interdigestive motor complex of normal subjects and patients with bacterial overgrowth of the small intestine. J Clin Invest 1977;59:1158-1166. 